\title{
The impact of social distancing during the COVID-19 pandemic on rates of pediatric idiopathic intussusception
}

\author{
Atsuhiko Handa ${ }^{1}$ (1) $\cdot$ Michael J. Callahan $^{1} \cdot$ Kosuke Kawai $^{2} \cdot$ Andy Tsai $^{1}$ \\ Received: 9 June 2021 / Revised: 23 August 2021 / Accepted: 16 September 2021 / Published online: 9 October 2021 \\ () The Author(s), under exclusive licence to Springer-Verlag GmbH Germany, part of Springer Nature 2021
}

\begin{abstract}
Background The causes of idiopathic ileocolic intussusception are unknown, with infection as the most likely culprit. Recently, social distancing measures were implemented during the coronavirus disease 2019 (COVID-19) pandemic to decrease transmissible infectious diseases, creating an opportune setting to study the role of infection on the pathogenesis of intussusception on a population level.

Objective To investigate the impact of social distancing on intussusception.

Materials and methods We retrospectively reviewed air contrast enemas and pylorus ultrasounds performed between March 2010 and March 2021 to identify cases of ileocolic intussusception and hypertrophic pyloric stenosis (HPS), using the latter as a negative control. The study time frame was divided into two periods: pre-pandemic (March 2010-February 2020) and pandemic (April 2020-March 2021). The number of cases that occurred in these two time periods were compared using the Poisson regression model.

Results Of the 407 cases of idiopathic ileocolic intussusception identified, 396 occurred in the pre-pandemic time period (mean $=39.6$ per 12 -month period) and 11 occurred in the 12 -month pandemic time period. The mean monthly number of intussusceptions showed a decline of $72 \%$ (95\% confidence interval [CI] 49-85\%) between the pre-pandemic and pandemic time periods ( 3.3 vs. 0.9 monthly cases; $P<0.001$ ). In contrast, the mean monthly number of HPS did not differ significantly $(P=0.19)$.

Conclusion Social distancing—imposed to curb the spread of infection during the COVID-19 pandemic—resulted in a significant decline in ileocolic intussusception, lending strong support to the theory that infection is the dominant cause of intussusception.
\end{abstract}

Keywords Children · COVID-19 · Hypertrophic pyloric stenosis $\cdot$ Intussusception $\cdot$ Pathogenesis $\cdot$ Ultrasound

\section{Introduction}

Intussusception is the telescoping of a proximal segment of the intestine (intussusceptum) into a more distal segment (intussuscipiens) and is a common cause of abdominal pain in children 6-36 months of age [1-8]. The incidence of intussusception is approximately 74/100,000 in children younger than 1 year of age, with the peak incidence among

Atsuhiko Handa

atsuhiko.handa@childrens.harvard.edu

1 Department of Radiology, Boston Children's Hospital, 300 Longwood Ave., Boston, MA 02115, USA

2 Institutional Centers for Clinical and Translational Research, Boston Children's Hospital and Harvard Medical School, Boston, MA, USA infants 5-7 months old [7]. Timely diagnosis and treatment are crucial because, if left untreated, intussusception can result in high morbidity and mortality. The current diagnostic approach includes ultrasound (US) with or without plain radiographs [2, 3, 9], and the treatment of choice at most institutions is fluoroscopic-guided air contrast enema [2, 4, 10-14]. Despite extensive research, the etiology of ileocolic intussusception remains largely unknown [3]. In a small percent of cases (2-12\%), a mass (such as Meckel diverticulum, polyp or enteric duplication cyst) acts as a pathological lead point $[4,6,15]$. Those remaining cases without a pathological lead point are considered idiopathic, even if there was a likely or possible triggering event such as infection [3]. Studies have shown a link between infection and intussusception [16-34]. Other speculative causes of 
intussusception include intestinal allergies, celiac disease, Crohn disease and certain anatomical variants [35].

Another relatively common condition affecting the gastrointestinal tracts in young children is hypertrophic pyloric stenosis (HPS), in which the pyloric muscle is abnormally thickened and elongated, resulting in near complete gastric outlet obstruction [36]. The etiology of this condition is also unknown; however, infection has not been implicated as the cause of HPS, a stark contrast to the current theory regarding the pathogenesis of ileocolic intussusception [37]. Although the speculative etiologies of HPS and intussusception are different, they do share two key commonalities: (1) both disease processes acutely affect the gastrointestinal tract requiring prompt diagnosis and intervention and (2) the peak age of incidence of both entities is during infancy (with HPS slightly younger).

The recent coronavirus disease 2019 (COVID-19) pandemic created an unprecedented situation where social distancing measures (such as the closures of schools and public places, physical distancing and the cancellation of mass gatherings) were implemented to reduce the transmission of severe acute respiratory syndrome coronavirus 2 (SARS-CoV-2) [38]. In the Commonwealth of Massachusetts, the governor declared a state of emergency on March 10, 2020 [39]. Kindergarten to 12th-grade schools and nonemergency childcare programs were closed in the same month, gradually reopening to in-person care after a year in April 2021. As a result of the mandated social distancing measures, there has been a global decline in the number of infectious diseases [40], including seasonal influenza [41], emergency department visits [42] and hospitalizations (for specific viral-associated conditions such as bronchiolitis and asthma) [43].

In this study, we investigate the role of infection as a cause of ileocolic intussusception. In contrast to virtually all other studies in the literature, where the goal was to establish a causal relationship between infection and intussusception, we intend to establish this relationship from an entirely different but complementary approach. Specifically, we conducted a retrospective observational study to show that the decrease in infections with social and physical distancing would result in a decrease in the number of intussusceptions. To establish this relationship, a large study cohort is required given the overall low incidence of this disease entity. Unintentionally and unknowingly, we have already engaged in such a study on a massive scale, specifically, at a population-level, during the COVID-19 pandemic. The widespread social distancing measures imposed for the past year curtailed the spread of infection, which we can leverage to study its impacts on intussusception by comparing the incidences of intussusception before and after the implementation of social and physical distancing measures. To strengthen this study, we added HPS as a negative control, given its similar disease profile and shared commonalities with childhood intussusception. In short, the objective of this study was to investigate the impact of social and physical distancing-implemented to curb the transmission of infections during the COVID-19 pandemic-on the incidence of ileocolic intussusception.

\section{Materials and methods}

This retrospective study was approved by the local institutional review board and was compliant with the Health Insurance Portability and Accountability Act. Informed consent was waived.

\section{Ileocolic intussusception study population}

An appropriate surrogate to identify children with clinically significant ileocolic intussusception is to identify those who underwent air contrast enema for intussusception reduction. At our hospital, the first step in the diagnosis of ileocolic intussusception is US, and the first line of treatment for this condition is via air contrast enema, including cases that ultimately require surgery. As a department, we do not perform liquid contrast enema or US-guided intussusception reductions.

We conducted a computerized search of the image database at our large tertiary children's hospital to identify air contrast enemas performed in children ( $\leq 18$ years old) between March 1, 2010, and March 31, 2021 (133 months). A pediatric radiologist (A.T.) with 11 years of post-fellowship experience reviewed the reports of these procedures. In cases where the air contrast enemas failed to reduce the intussusception and required surgical intervention, the surgical reports were reviewed. Exclusion criteria were as follows: (1) previous air contrast enema(s) within the past year, (2) surgically proven pathological lead point as the cause of intussusception and (3) intussusception that did not involve the colon. The purpose of exclusion criterion 1 was to eliminate children with multiple air contrast enemas performed for intussusception recurrence and/or treatment failures. The generous 1-year time window specified for this exclusion criterion was to avoid double counting during the data analysis. To stay within the scope of this investigation, exclusion criteria 2 and 3 were implemented to ensure that our study cohort included only those with idiopathic intussusceptions (by excluding those with surgically proven pathological lead points) and those that are ileocolic in location, respectively.

\section{Hypertrophic pyloric stenosis study population}

A computerized search of the image database at our large tertiary children's hospital was similarly conducted to 
Fig. 1 A flowchart illustrates the identification of patients for inclusion in the ileocolic intussusception study cohort

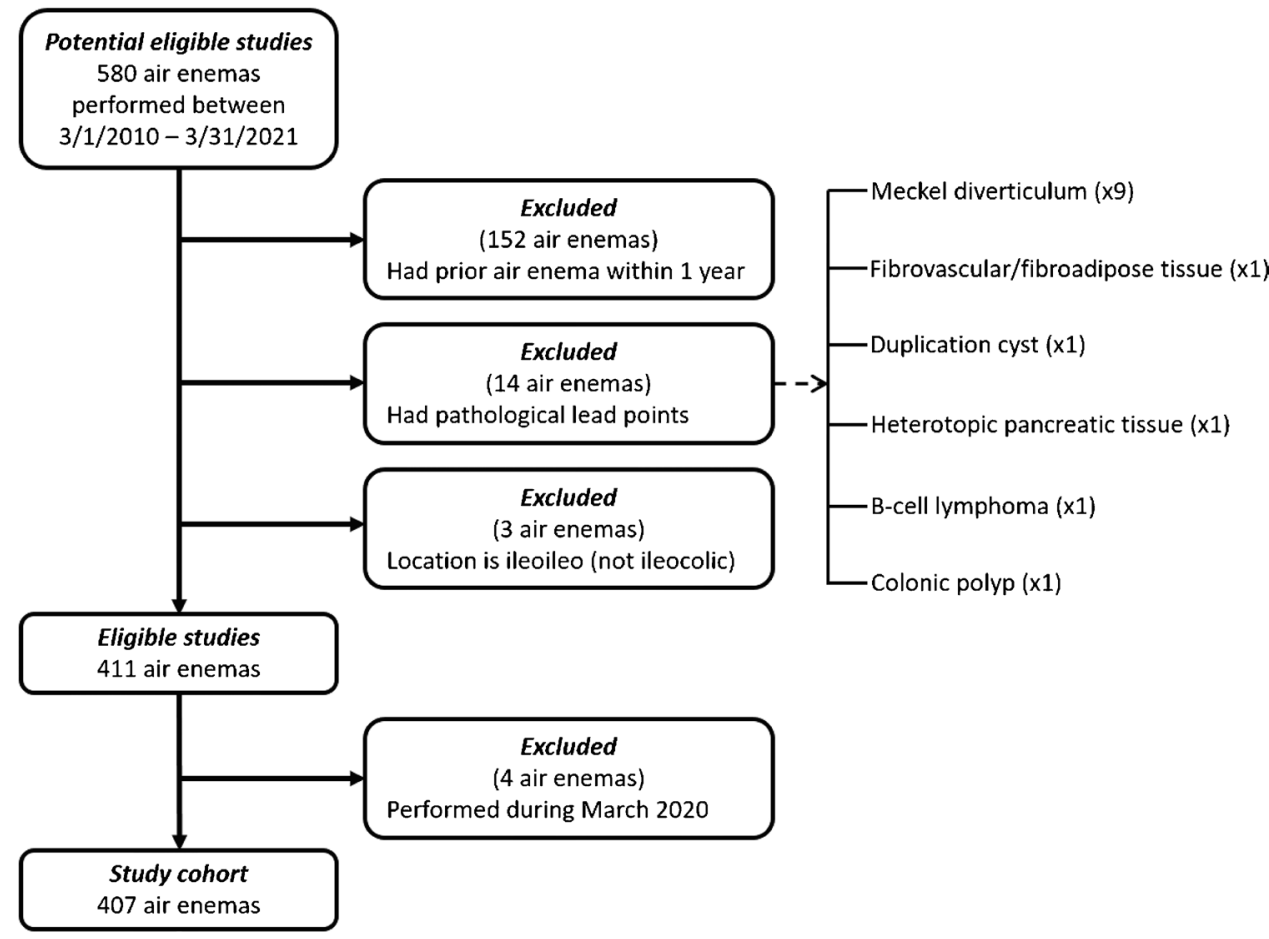

identify pylorus US exams performed during early infancy ( $\leq 6$ months old) between March 1, 2010, and March 31, 2021 (the same $\sim 11$ years). The same pediatric radiologist reviewed the reports of these US exams to identify those with a diagnosis of HPS. The exclusion criterion was a previous positive pylorus US to avoid double counting.

\section{Pre-pandemic and pandemic time periods}

This investigation occurred between March 1, 2010, and March 31, 2021. To test our study hypothesis, this period of time was separated into two subsets: pre-pandemic and pandemic. Because the regional pandemic public health response was initiated during March 2020, part of this month was under mandated social and physical distancing. As a result, March 2020 was considered a transitional month, and it was excluded from the study. The pre-pandemic period consisted of the 120 months before the initiation of the social distancing (i.e. March 1, 2010-February 29, 2020). In contrast, the pandemic period consisted of the 12 months following the initiation of social distancing (i.e. April 1, 2020-March 31, 2021).

\section{Statistical analysis}

We compared the monthly number of patients with ileocolic intussusceptions and HPS diagnosed during the pre-pandemic time period (120 months) against those diagnosed during the pandemic time period (12 months) using the Poisson regression model with log link function. To compare the data over a longer period of time, we divided the 120 months before the initiation of social distancing into 10 contiguous 12-month intervals. Each time interval extends from March 1 of 1 year to February 28 (or February 29 during leap years) of the following year. The 12 months following the initiation of social distancing were grouped together as one. This partitioning scheme resulted in 10 groupings in the pre-pandemic period and 1 grouping in the pandemic period, applied to both the ileocolic intussusception and HPS study cohorts. For each study cohort, the number of patients in each of the eleven 12-month groupings were tabulated, then the numbers in the pre-pandemic period were compared to those in the pandemic period (using mean and standard deviation [SD] as the summary statistics for comparison). The seasonal variation of ileocolic intussusception was evaluated by examining case counts by month. We examined any monthly variation in cases using a $\chi^{2}$ test. All analyses were conducted with a statistical significance threshold of $P<0.05$. Statistical analysis was conducted using SAS version 9.4 (SAS Institute, Inc., Cary, NC). 
Fig. 2 The number of patients diagnosed with ileocolic intussusception (a) and hypertrophic pyloric stenosis (b) over the

132-month study interval, excluding March 2020. Black bars correspond to the pre-pandemic time period (i.e. the 10 groups covering the 120 months before the pandemic social distancing measures). The white bar corresponds to the pandemic time period (i.e. the 12-month time interval after the initiation of the pandemic social distancing measures)

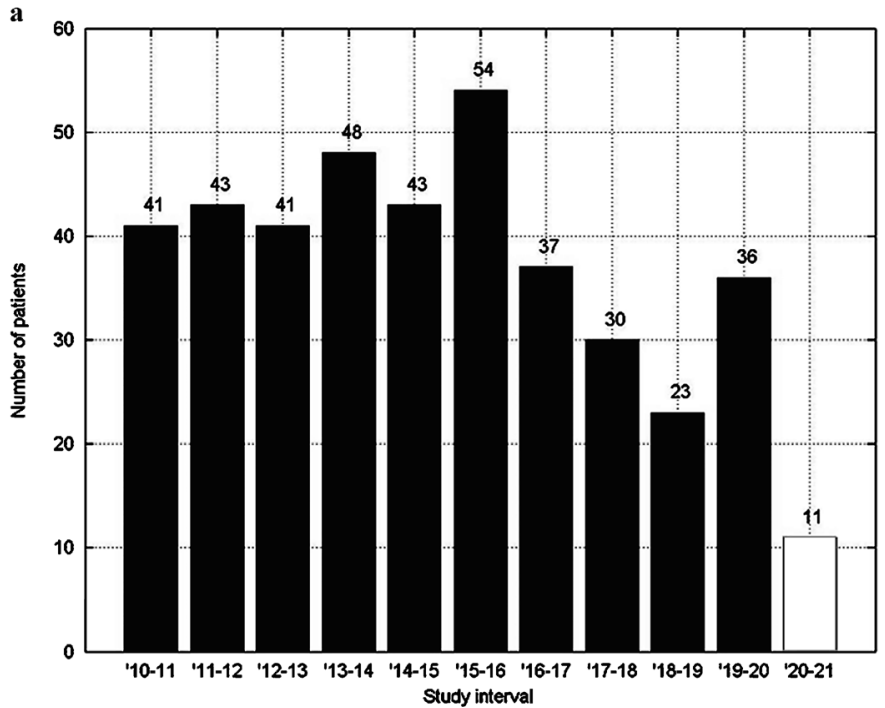

b

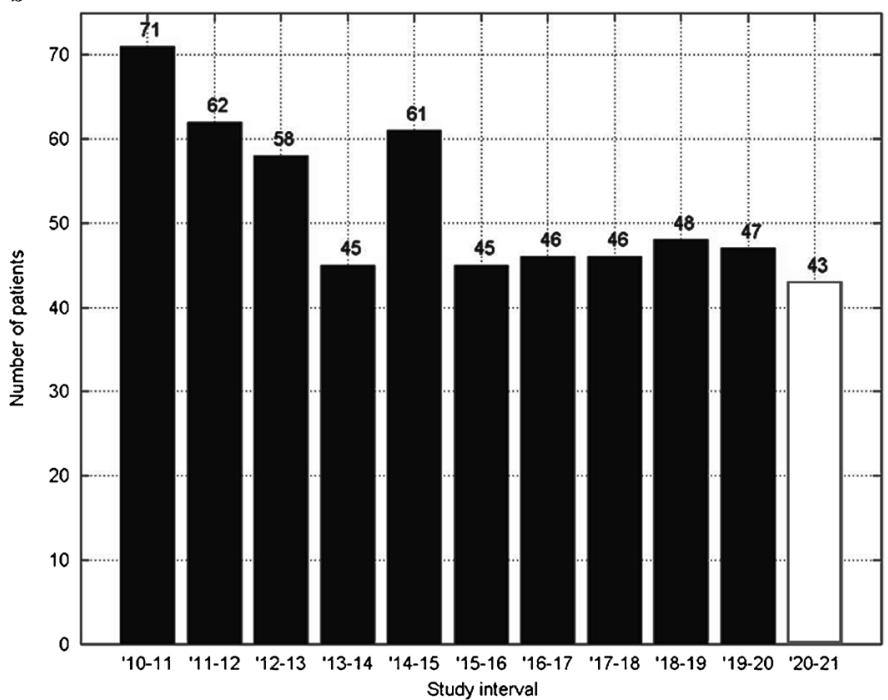

Of the 407 ileocolic intussusceptions that occurred during the study, 396 occurred in the pre-pandemic time period (mean $=39.6$ and $\mathrm{SD}=8.8$ per 12 -month period). The 11 intussusceptions that occurred in the 12-month pandemic time period was a decline of $72 \%$ from the pre-pandemic average (Fig. 2). Only one patient in this cohort was noted to be COVID-19 positive (13 days before the diagnosis of intussusception). Monthly, the mean number of ileocolic intussusceptions was $3.3(\mathrm{SD}=1.9)$ during the pre-pandemic time period and $0.9(\mathrm{SD}=1.0)$ during the pandemic time period. During the pandemic time period, the monthly number of intussusceptions declined by $72 \%$ (95\% confidence interval [CI] 49-85\%; $P<0.001$; Table 1). A more in-depth analysis showed that the decline in monthly number of cases between the two time periods was observed among males, among females, among children $<2$ years old and among children 2-4 years old. We found no evidence of seasonality of intussusception $(P=0.74)$. 


\section{Hypertrophic pyloric stenosis study cohort}

For the HPS study cohort, 3,830 pylorus US exams were performed during the same study period, and 572 patients were diagnosed with HPS (481 males, 91 females; mean age $=1.3$ months, $\mathrm{SD}=0.7$ months, range $=0.36-5.0$ months). Of the 572 patients, 529 were diagnosed in the pre-pandemic time period $($ mean $=52.9$ and $\mathrm{SD}=9.3$ per 12 -month period) and 43 patients were diagnosed in the 12-month pandemic time period (Fig. 2). Monthly, the mean number of HPS cases diagnosed did not differ between the pre-pandemic time period (mean $=4.4$, $\mathrm{SD}=2.3)$ and the pandemic time period (mean $=3.6$, $\mathrm{SD}=2.5 ; P=0.19)$.

\section{Discussion}

We described the incidence of ileocolic intussusception during the pre-pandemic and pandemic periods at a large tertiary children's hospital, both on a yearly basis and on a monthly basis, over the past 11 years. The epidemiological characteristics of children with intussusception in our study recapitulated previous studies, including the age distribution, pathological lead points and those in the ileocolic regions. The lack of seasonal variation also confirms findings described previously $[5,7]$. The number of intussusception cases that occurred in the pandemic time period showed a statistically significant decline of $72 \%$ as compared to the pre-pandemic period. In contrast, the incidence of HPS remained steady.

The significant decrease in the incidence of ileocolic intussusception during the COVID-19 pandemic public health response supports our hypothesis that transmissible infection is a major factor in causing ileocolic intussusception. We speculate that mandatory social and physical distancing decreased the rate of transmissible infections, which likely reduced infection-triggered bowel inflammation and lymphadenopathy and in turn decreased the incidence of idiopathic ileocolic intussusception. Furthermore, the 72\% decrease in the incidence of ileocolic intussusception from the pre-pandemic to the pandemic time periods suggests that infection probably constitutes a larger portion of cases than previously reported (approximately 30\%) [5]. In sum, our data support previous studies that demonstrated a causal relationship between infectious agents and ileocolic intussusception [16-32].

A few published reports describe ileocolic intussusception rates related to the COVID-19 pandemic [44-46]. We note a recent letter to the editor reporting a decrease in pediatric intussusception cases during the COVID-19 pandemic, with the purpose of dispelling the myth that
COVID-19 may be causing an increased number of ileocolic intussusceptions [47]. In our study, during the pandemic period, only $1 / 11$ patients with intussusception was documented to have active COVID-19 infection. Given these data, we postulate that COVID-19-related ileocolic intussusception is uncommon, and other infectious agents constitute the majority of infectious predisposition.

In contrast to all the previous studies showing infection as a risk factor for intussusception, our study set out to support this causation from a different angle. Specifically, we utilized statistical inference and the widespread pandemic public health response to support the notion that a lack of infection is a safeguard against ileocolic intussusception. Although our approach differed from that of others, the results were nevertheless valid and complementary, further strengthening the theory of infection as the main cause of intussusception.

The use of HPS as a negative control in our study strengthened our results. We believe that HPS served as a satisfactory negative control for three primary reasons. Firstly, this condition closely mirrored intussusception in many respects (age of presentation, acuity of condition, unknown pathogenesis and organ system involvement), with the major difference being that infection has not been implicated as a potential etiology of HPS. Secondly, the epidemiological characteristics of our HPS study population mirrored those reported previously, providing firm scientific grounds and support as a negative control. Thirdly, we argue that the lack of change in the incidence of HPS in the pre-pandemic period and pandemic period is a strong foundation to address possible study confounders and biases that may have emerged during the pandemic (such as alterations in referral pattern, differences in health care access and changes in diagnostic work-up).

The decrease in the incidence of intussusception posed an educational challenge to radiology residency and fellowship programs during the pandemic. The number of cases during the pandemic was approximately one-fourth of the pre-pandemic level, substantially limiting our trainees' valuable experiences and competence in performing air contrast enema procedures. Additional measures or remediation may be necessary for trainees to become comfortable in performing such an important therapeutic procedure.

Our study has limitations, including its retrospective nature. This study was limited by the small number of ileocolic intussusceptions diagnosed during the pandemic period. A multi-institutional study would be required to increase this number and further strengthen our findings. It was not possible to calculate the incidence rate because of the difficulty in defining the source population for this hospital-based study. Another limitation was the potential development of differential referral bias, suboptimal health 
Table 1 Monthly number of ileocolic intussusceptions and hypertrophic pyloric stenoses during the pre-pandemic (March 1, 2010, to February 29, 2020) and pandemic (April 1, 2020, to March 31, 2021) time periods

\begin{tabular}{llll}
\hline & $\begin{array}{l}\text { Pre-pandemic: monthly number of } \\
\text { cases, mean (SD) }\end{array}$ & $\begin{array}{l}\text { Pandemic: monthly number of } \\
\text { cases, mean (SD) }\end{array}$ & $\begin{array}{l}\text { Ratio of event counts } \\
(95 \% \text { CI })\end{array}$ \\
\hline $\begin{array}{l}\text { Ileocolic intussusception } \\
\text { All children }\end{array}$ & $3.3(1.9)$ & $0.9(1.0)$ & $0.28(0.15,0.51)$ \\
By gender & & & $0.27(0.13,0.56)$ \\
$\quad$ Male & $2.2(1.5)$ & $0.6(0.8)$ & $0.30(0.11,0.81)$ \\
Female & $1.1(1.0)$ & $0.3(0.5)$ & 0.0018 \\
By age category & & $0.7(0.7)$ & $0.40(0.20,0.82)$ \\
$\quad<2$ y & $1.7(1.3)$ & $0.2(0.6)$ & $0.21(0.07,0.65)$ \\
2 y to $<4$ y & $1.2(1.1)$ & 0 & 0.012 \\
4 y to $\leq 18$ y & $0.4(0.7)$ & $3.6(2.5)$ & 0.007 \\
Hypertrophic pyloric stenosis & & & $0.81(0.58,1.09)$ \\
All children & $4.4(2.3)$ & 0.19 \\
\hline
\end{tabular}

$C I$ confidence interval, $S D$ standard deviation, $y$ years

care access and change in diagnostic work-up during the pandemic. Some may argue that parents may be more reluctant to seek care, telehealth may present as a barrier to timely and accurate diagnosis, and hospital access may be more restricted. However, these were unlikely major contributors given the unchanged incidence of HPS, a condition that has a similar disease profile as intussusception. In addition, ileocolic intussusception commonly presents as an urgent clinical situation, requiring immediate therapy. Lastly, we did not review every single patient's chart for antecedent illness or any microbial result. Thus, it would be impossible to know how many of our patients truly had an antecedent infectious disease. Such meticulous and time-consuming study may clarify the direct causal linkage on a large population scale.

\section{Conclusion}

The COVID-19 pandemic created an unprecedented time of social and physical distancing for children and their siblings, immediate family, and caregivers, which served to substantially reduce not only COVID-19 infections but also other transmissible infectious diseases. This unique isolating environment resulted in a statistically significant decline in the incidence of ileocolic intussusception, but an unchanged incidence of HPS at our institution, lending support to the theory that infectious etiology is a dominant cause of intussusception. Future data from the post-pandemic period (once social distancing measures have been completely lifted) with demonstration of a rebound in the number of intussusceptions would further strengthen our results.

\section{Declarations}

Conflict of interest None.

\section{References}

1. Perrin WS, Lindsay EC (1921) Intussusception: a monograph based on 400 cases. Br J Surg 9:46-71

2. del-Pozo G, Albillos JC, Tejedor D et al (1999) Intussusception in children: current concepts in diagnosis and enema reduction. Radiographics 19:299-319

3. Edwards EA, Pigg N, Courtier J et al (2017) Intussusception: past, present and future. Pediatr Radiol 47:1101-1108

4. Ma GMY, Lillehei C, Callahan MJ (2020) Air contrast enema reduction of single and recurrent ileocolic intussusceptions in children: patterns, management and outcomes. Pediatr Radiol 50:664-672

5. Buettcher M, Baer G, Bonhoeffer J et al (2007) Three-year surveillance of intussusception in children in Switzerland. Pediatrics 120:473-480

6. Stringer MD, Pablot SM, Brereton RJ (1992) Paediatric intussusception. Br J Surg 79:867-876

7. Jiang J, Jiang B, Parashar U et al (2013) Childhood intussusception: a literature review. PLoS ONE 8:e68482

8. Daneman A, Alton DJ (1996) Intussusception. Issues and controversies related to diagnosis and reduction. Radiol Clin N Am 34:743-756

9. Binkovitz LA, Kolbe AB, Orth RC et al (2019) Pediatric ileocolic intussusception: new observations and unexpected implications. Pediatr Radiol 49:76-81

10. Kaplan SL, Magill D, Felice MA et al (2017) Intussusception reduction: effect of air vs. liquid enema on radiation dose. Pediatr Radiol 47:1471-1476

11. Khong PL, Peh WC, Lam CH et al (2000) Ultrasound-guided hydrostatic reduction of childhood intussusception: technique and demonstration. Radiographics 20:E1

12. Khanna G, Applegate K (2008) Ultrasound guided intussusception reduction: are we there yet? Abdom Imaging 33:38-40 
13. Cohen MD (2002) From air to barium and back to air reduction of intussusception in children. Pediatr Radiol 32:74

14. Sadigh G, Zou KH, Razavi SA et al (2015) Meta-analysis of air versus liquid enema for intussusception reduction in children. Am J Roentgenol 205:W542-549

15. Daneman A, Alton DJ, Lobo E et al (1998) Patterns of recurrence of intussusception in children: a 17-year review. Pediatr Radiol 28:913-919

16. Nicolas JC, Ingrand D, Fortier B, Bricout F (1982) A one-year virological survey of acute intussusception in childhood. J Med Virol 9:267-271

17. Nylund CM, Denson LA, Noel JM (2010) Bacterial enteritis as a risk factor for childhood intussusception: a retrospective cohort study. J Pediatr 156:761-765

18. Lin Z, Cohen P, Nissan A et al (1998) Bacterial wall lipopolysaccharide as a cause of intussusception in mice. J Pediatr Gastroenterol Nutr 27:301-305

19. Bhisitkul DM, Todd KM, Listernick R (1992) Adenovirus infection and childhood intussusception. Am J Dis Child 146:1331-1333

20. Hsu HY, Kao CL, Huang LM et al (1998) Viral etiology of intussusception in Taiwanese childhood. Pediatr Infect Dis J $17: 893-898$

21. Bines JE, Liem NT, Justice FA et al (2006) Risk factors for intussusception in infants in Vietnam and Australia: adenovirus implicated, but not rotavirus. J Pediatr 149:452-460

22. Zupancic JA, Pennie RA, Issenman R (1994) Intussusception in a child with cytomegalovirus infection. Pediatr Infect Dis J 13:548-549

23. Konno T, Suzuki H, Kutsuzawa T et al (1978) Human rotavirus infection in infants and young children with intussusception. $\mathrm{J}$ Med Virol 2:265-269

24. Burchfield DJ, Rawlings D, Hamrick HJ (1983) Intussusception associated with Yersinia enterocolitica gastroenteritis. Am J Dis Child 137:803-804

25. Hervas JA, Alberti P, Bregante JI et al (1992) Chronic intussusception associated with Yersinia enterocolitica mesenteric adenitis. J Pediatr Surg 27:1591-1592

26. Winesett MP, Pietsch JB, Barnard JA (1996) Yersinia enterocolitica in a child with intussusception. J Pediatr Gastroenterol Nutr 23:77-80

27. Hansen MG, Pearl G, Levy M (2001) Intussusception due to Yersinia enterocolitica enterocolitis in a patient with betathalassemia. Arch Pathol Lab Med 125:1486-1488

28. Staatz G, Alzen G, Heimann G (1998) Intestinal infection, the most frequent cause of invagination in childhood: results of a 10-year clinical study. Klin Padiatr 210:61-64

29. Georgoula C, Ward HC (2004) Recurrent intussusception associated with Escherichia coli 0157 infection. Dig Liver Dis 36:557

30. Fitzgerald J, Troncone R, Cole CR, Kaul A (2001) Clinical quiz. An ileocolonic intussusception associated with $C$. difficile infection. J Pediatr Gastroenterol Nutr 33:289, 300

31. Matsushita M, Suzaki T, Hajiro K (1994) Intussusception associated with Salmonella typhimurium enterocolitis. Am J Gastroenterol 89:1246-1248
32. Raveenthiran V, Rao PV, Murugesan M (2005) Intussusception complicating typhoid fever. J R Soc Med 98:368-369

33. Murphy TV, Gargiullo PM, Massoudi MS et al (2001) Intussusception among infants given an oral rotavirus vaccine. $\mathrm{N}$ Engl $\mathrm{J}$ Med 344:564-572

34. Centers for Disease Control and Prevention (CDC) (1999) Withdrawal of rotavirus vaccine recommendation. Morb Mortal Wkly Rep 48:1007

35. Marsicovetere P, Ivatury SJ, White B, Holubar SD (2017) Intestinal intussusception: etiology, diagnosis, and treatment. Clin Colon Rectal Surg 30:30-39

36. Blumhagen JD, Maclin L, Krauter D et al (1988) Sonographic diagnosis of hypertrophic pyloric stenosis. Am J Roentgenol 150:1367-1370

37. Galea R, Said E (2018) Infantile hypertrophic pyloric stenosis: an epidemiological review. Neonatal Netw 37:197-204

38. Grohskopf LA, Sokolow LZ, Broder KR et al (2017) Prevention and control of seasonal influenza with vaccines: recommendations of the Advisory Committee on Immunization PracticesUnited States, 2017-18 influenza season. MMWR Recomm Rep $66: 1-20$

39. Commonwealth of Massachusetts (2021) COVID-19 state of emergency. https://www.mass.gov/info-details/covid-19-stateof-emergency. Accessed 5 June 2021

40. Hatoun J, Correa ET, Donahue SMA, Vernacchio L (2020) Social distancing for COVID-19 and diagnoses of other infectious diseases in children. Pediatrics 146:e2020006460

41. Lee H, Lee H, Song K-H et al (2021) Impact of public health interventions on seasonal influenza activity during the COVID-19 outbreak in Korea. Clin Infect Dis 73:e132-e140

42. Raucci U, Musolino AM, Di Lallo D et al (2021) Impact of the COVID-19 pandemic on the emergency department of a tertiary children's hospital. Ital J Pediatr 47:21

43. Wilder JL, Parsons CR, Growdon AS et al (2020) Pediatric hospitalizations during the COVID-19 pandemic. Pediatrics 146:e2020005983

44. Makrinioti H, MacDonald A, Lu X et al (2020) Intussusception in 2 children with severe acute respiratory syndrome coronavirus-2 infection. J Pediatr Infect Dis Soc 9:504-506

45. Bazuaye-Ekwuyasi EA, Camacho AC, Saenz Rios F et al (2020) Intussusception in a child with COVID-19 in the USA. Emerg Radiol 27:761-764

46. Osorno JF, Giraldo M, Marin AF, Figueroa LM (2021) Novel coronavirus infection in an infant with intussusception. Glob Pediatr Health 8:2333794X211012978

47. Zheng J, Ye Y, Liao Y, Wang B (2020) Fewer paediatric intussusception cases during the COVID-19 pandemic. J Paediatr Child Health 56:1165

Publisher's note Springer Nature remains neutral with regard to jurisdictional claims in published maps and institutional affiliations. 